\title{
Notas comparativas de inmigrantes chinos y japoneses*
}

Recibido: $19 / 02 / 2020$

Aprobado: 20/05/2020

Publicado: 25/08/2020

Humberto Rodríguez Pastor

Universidad Nacional Mayor de San Marcos <hrodriguez2541937@hotmail.com>

\section{RESUMEN}

Visión panorámica de los complejos procesos migratorios de dos grupos de trabajadores asiáticos inmigrados, chinos y japoneses, que llegaron a Perú en diferentes períodos. Estos fueron casi continuos, ya que su presencia respondía a la necesidad de mano de obra en las haciendas costeñas y que se enfrentaron a formas coercitivas de exigencias. Por eso, la fuga fue semejante en ambos casos.

Palabras Clave: Chinos, japoneses, inmigración, Perú, trabajadores.

\section{Comparative notes regarding chinese and japanese immigrants}

\begin{abstract}
Panoramic view of the complex migratory processes of two groups of migrant Asian workers, Chinese and Japanese, who arrived in Peru in different periods. These were almost continuous, since their presence responded to the need for labor on the coastal haciendas and that they faced coercive forms of demands. Therefore, the leak was similar in both cases.
\end{abstract}

KeYwords: Chineses, japaneses, Peru, inmigration, workers.

* Estas notas se apoyan en la revisión de un artículo previamente publicado por el autor (Rodríguez Pastor, 2007) bajo convenio entre la Fundación San Marcos de la Universidad Nacional Mayor de San Marcos y el Japanese American National Museum, Proyecto Discover Nikkei. 


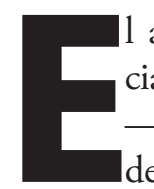
asiático chino, por lo general de la provincia sureña de Kuangtong, que masivamente -aproximadamente 92.000, casi la totalidad de ellos fueron hombres - llegó durante el período 1849-1874 fue destinado principalmente al trabajo agrícola en casi todas las haciendas de la costa peruana (con excepción de las piuranas), pero también se le condujo a las labores en las islas guaneras, a la construcción de ferrocarriles y como servidumbre doméstica en las ciudades, principalmente en Lima y Callao.

A mediados del siglo XIX esa agricultura costeña de exportación — principalmente la dedicada a la caña de azúcar y algodón- ingresaba con éxito a un mercado internacional cuyos buenos precios permitió una notable rentabilidad, y por esta razón una gran capitalización en las grandes propiedades agrícolas y, a su vez, su modernización y mecanización. Socialmente, esta riqueza condujo a la aparición de un sector pudiente que perduró, con poder político y social, hasta la reforma agraria de 1969, durante el gobierno del general Juan Velasco Alvarado.

Cincuenta ańos después que comenzó el arribo de chinos al Perú, se inició, en 1899, la llegada de japoneses, migración que duró, aunque en años discontinuos, veinticuatro ańos, hasta 1923. Estos inmigrantes, que en número aproximado fueron 18.500, - $89 \%$ de sexo masculino-, eran originarios de las prefecturas de Niigata, Yamaguchi e Hiroshima, y también una cantidad mayor de Okinawa.

Estos nuevos asiáticos en territorio peruano fueron destinados en su totalidad a las labores agrícolas de algunas de las haciendas costeńas, principalmente las del departamento de Lima (valles del Rímac, Cañete, Pativilca y Supe) que estaban dedicadas al algodón y a la caña de azúcar. El interés mayor de los propietarios de estas haciendas era obtener mano de obra estable (Rodríguez, 2007).

En estas primeras décadas del siglo XX ya descendían de la sierra para trabajar en la agricultura del litoral miles peones que eran tentados a través del enganche; se encontraban, pues, adeudados por el pago adelantado que recibían en sus lugares de origen, no obstante, su presencia no era estable, regresaban a la serranía, donde por lo general tenían tierras de cultivo. La cańa y el algodón, por entonces, requería trabajo seguro ininterrumpido, pues desde los chinos culíes ya era parte de los engranajes de la comercialización en el mercado interncional; el peón japonés aseguró esta continuidad.

\section{Realidades de viajes diferentes}

Fueron momentos históricos diferentes por los que pasaron los viajes que hicieron chinos y japoneses que trabajaron en la agricultura peruana. Los 92.000 chinos fueron trasladados en un total de 247 viajes, lo que nos da un promedio de 369 por cada viaje; los 18.500 japoneses que se trasladaron a Perú lo hicieron en 82 viajes, es decir, un promedio de 225 personas en cada viaje.

El traslado de los miles de culíes era un período sucessivo a los años en que se trasportaba a los esclavos negros africanos a América, aunque las condiciones eran bastante similares: al igual que a los esclavos africanos, los semiesclavos culíes eran colocados (hacinados) en el entrepuente con muy poco espacio para cada uno, falta de higiene, mala comida y peor agua para beber, lo que producía enfermedades estomacales que los llevaba a la muerte; por este motivo, no menos del $10 \%$ del total de los que salieron de China fallecieron antes de llegar a Perú. Las naves en que se hicieron todos estos viajes eran fragatas y barcas a vela con no más de 1600 toneladas de registro, que en promedio demoraban 120 días en cruzar el océano Pacífico.

Desde el primer barco en el que llegaron japoneses este medio de trasporte marítimo era algo totalmente diferente. En menos de cincuenta años, los barcos de vapor habían desplazado a los barcos a vela. Es así que el histórico Sakura Maru tenía 2.953 toneladas de registros y hasta satisfacía la costumbre de los primeros inmigrantes japoneses de bañarse en agua caliente (Rodríguez, 2007).

\section{Diferencias en las condiciones de trabajo diferentes}

Los miles de chinos culíes que salieron de los puertos de la China imperial solo pudieron hacerlo después de haber firmado de manera individual artificiosos contratos. Esto era así en cuanto la prohibición de la esclavitud y de la trata esclava en el mundo era ya una política aceptada y controlada por las grandes potencias económicas de entonces, principalmente Inglaterra. Un contrato como los que firmaban los chinos representaba la decisión personal de trasladarse a trabajar a otros continentes de manera voluntaria. Eso ya no era considerado como esclavitud. 
A nuestro criterio, la condición laboral del chino fue la de semiesclavo por contrato. Esto significaba la obligación del culí de trabajar para un amo o patrón —que en el Perú aún mantenía una mentalidad esclavista-, en lo que él determinara durante ocho años (aunque los primeros años fue de solo cinco ańos) $y$, a continuación, quedaba libre si es que no aceptaba recontratarse. Mientras duraran esos años obligatorios, el patrón podía permutar o transferir a otro amo al trabajador migrante asiático. De su parte, la aceptación de recontratarse era, eso sí, casi un acto voluntario del trabajador oriental.

Por este mismo motivo, a pesar de que habían transcurrido algunas décadas, la manera legal e internacionalmente posible para la emigración de trabajadores desde Japón requería la aquiescencia - firma de por medio- de cada emigrante y hasta del permiso del Estado imperial japonés.

La diferencia más importante del emigrante chino o japonés era la "pertenencia" formal de uno $\mathrm{u}$ otro ante una tercera persona jurídica. De manera general, los chinos semiesclavos físicamente eran traspasados al venderse el contrato a quienes veían que les convenía las condiciones de las cláusulas. En su mayoría los interesados fueron hacendados. Por su parte, los japoneses eran «propiedad» de la casa enganchadora (hubo varias) hasta que cumplían el término del tiempo obligatorio que tenían que trabajar para la empresa que suscribía el contrato. Una hacienda, por ejemplo, firmó varios contratos con la Casa Morioka, y ésta para asegurarse cumplimiento y poder reclamar exigía que los emigrantes firmaran con ella contratos iguales en contenido, punto por punto, que el primero. Pero las exigencias también eran en sentido contrario: los trabajadores podían hacer sus reclamos a la Morioka y ésta a la Sociedad, siempre que hubiera incumplimiento.

$\mathrm{Y}$ en tanto perduraba el trato exigente y brutal, así como la incomprensión cultural, los chinos respondieron a esta agresión de diferentes maneras: lo más frecuente fueron las fugas o cimarronaje (tanto en ciudades como en el campo), tumultos localizados (en general en las haciendas por maltratos físicos o exigencias excesivas de trabajo), asesinatos a dueños de las propiedades agrícolas o a sus empleados, rebeliones (la más importante fue en el valle de Pativilca, en el mes de septiembre de 1870), así como la autoeliminación.
Su condición era diferente. Los contratos que también hubo no eran personales, se hicieron a través de grandes compañías - la más importante fue la Morioka- y ellas, a su vez, hacían un contrato con cada emigrante facilitándole, al momento de la firma, una cantidad de dinero o enganche. El control y cumplimiento del trabajo cotidiano se realizaba con empleados de las haciendas y con personal de las compañías. Según esos contratos, éstas debían mantener un número fijo de trabajadores en el trabajo de las propiedades agrícolas. Las compañías ganaban una proporción por cada día de trabajo del bracero emigrado. En todos estos acuerdos no estaban al margen ni el Estado japonés ni dejaban de estar vigilantes los representantes diplomáticos de ese Estado en el Perú.

En suma, al peón japonés se le debe considerar como un trabajador emigrante enganchado en su país por compañías negociantes en este rubro con el consentimiento del estado japonés (Rodríguez, 2007).

\section{Epílogo}

A pesar de tratarse de trabajadores chinos y japoneses que estuvieron en condiciones diferentes en las haciendas costeñas peruanas y en distintos tiempos históricos, hubo reacciones similares entre ellos - aunque no iguales - en tanto en todo momento fueron invariables tanto el trato exigente y brutal como la incomprensión cultural. Frente a esta agresión, hubo diversas reacciones comunes entre estos trabajadores: las más frecuentes fueron las fugas y el cimarronaje, pero también tumultos localizados.

\section{Referencias bibliográficas}

Arona, Juan de (1972). La inmigración en el Perú. Academia Diplomática del Perú, Lima.

BASAdre, Jorge (1969). Historia de la República del Perú. Ts. I-XVI, Editorial Universitaria, Lima, Gta edic.

Benvenutto Murietta, Pedro M. (1983). Quince plazuelas, una alameda y un callejón. Fondo del Libro Banco Industrial, Lima.

Borja, César (1877). La inmigración china es un mal necesario de evitar. En: Anales Universitarios del Perú. T. X, Lima, 1877, pp. 47-92. 
Camino Calderón, Carlos (1945). Diccionario folklórico del Perú. Cía. de Impresiones y Publicidad, Lima.

Castro de Mendoza, Mario (1980). La marina mercante de la República, 1821-1968. Concytec, Lima.

Castro Sampaio, Manuel de (1867). Os chins de Macau. Hongkong, Tipografía de Noronha e Filhos.

Choy, Emilio (1965). La esclavitud de los chinos en el Perú. En: Tareas del Pensamiento Peruano. № 8, junio, pp. 45-53.

Dañino Ribatto, Guillermo (1990). Desde China. Un país fascinante y misterioso. Pontificia Universidad Católica del Perú y Editorial Labrusa, Lima.

Dañino Ribatto, Guillermo (2013). Enciclopedia de la cultura china. Ediciones en Lenguas Extranjeras Cía. Limitada, Beijing, China.

Dañino Ribatto, Guillermo (2014). Religiones en China. En: China y Perú en el arte y la cultura. Instituto. Confucio, Universidad Ricardo Palma, Lima. pp. 32-68.

Do Inso, Jaime (Capitao tenente) (1930). Macao. A Mais Antiga Colonia Europeia no Extremo Oriente. Macao.

Fuentes, Manuel Atanasio (1988). Lima Apuntes históricos, descriptivos, estadísticos y de costumbres. Fondo del Libro. Banco Industrial del Perú.

Fuкumoto Sato, Mary Nancy (1997). Hacia un nuevo sol. Japoneses y sus descendientes en el Perú. Historia, cultura e identidad. Asociación Peruano Japonesa del Perú, Lima.

Gonzales, Michael J. (1974). Cayalti: The formation of a rural proletariat on a peruvian sugar cane plantation, 1875-1933. Tesis para optar grado de Doctor of Philosophy de la Universidad de California, Berkeley.

Hu-De Hart, Evelyn (1988). Chinos comerciantes en el Perú: breve y preliminar bosquejo histórico (1869-1924). En: Primer Seminario sobre Poblaciones Inmigrantes. T. II. pp. 127-135.

IIDA, Juan Kazuo (1999). Los primeros inmigrantes. Arte y Proyecto Gráfico, Lima.

Iто, Luis Tsutomi (2014). Andando 75 años por los caminos del Perú. La inmigración japonesa 1899-1974). Traducción al español de Carmen Chieko de Noguchi. Editorial Perú Shimpo S.A., Lima.

Iwasaki Cauti, Fernando (1989). Extremo Oriente y Perú en el siglo XVI. Editorial Mapfre S.A. Madrid, España.

Lausent-Herrera, Isabelle (1990). Pasado y presente de la comunidad japonesa en el Perú. Colección Mínima, Instituto de Estudios Peruanos.

Lausent-Herrera, Isabelle (2000). Sociedades y templos chinos en el Perú. Fondo Editorial del Congreso del Perú, Lima.
Macera, Pablo (1977). Las plantaciones azucareras en el Perú, 1821-1875. En: Trabajos de Historia. T. IV Instituto Nacional de Cultura, Lima.

Mayer de Zulen, Dora (1924). La China silenciosa y elocuente. Editorial Renovación, Lima.

McKeown, Adam (1996). Inmigración china al Perú, 1904-1937; Exclusión y negociación. En: Histórica, Vol. XX No1, julio.

McKeown, Adam (2001). Chinese Migrant Networks and Cultural Change: Peru, Chicago, Hawaii, 1900-1936. Chicago: The University of Chicago Press.

Morimoto, Amelia (1979). Los inmigrantes japoneses en el Perú. Taller de Estudios Andinos, Universidad Nacional Agraria, Lima.

Rocca Torres, Luis (1997). Japoneses bajo el Sol de Lambayeque. Coedición Universidad Nacional Pedro Ruiz Gallo y Asociación Peruano Japonesa del Perú, Lambayeque y Lima.

Rodríguez Pastor, Humberto (1989). Hijos del Celeste Imperio en el Perú (1850-1900). Migración, agricultura, mentalidad y explotación. Instituto de Apoyo Agrario, Lima.

Rodríguez Pastor, Humberto (2000). Herederos del Dragón. Historia de la comunidad china en el Perú. Fondo Editorial del Congreso del Perú, Lima.

Rodríguez Pastor, Humberto (11 de septiembre de 2007). Algunas comparaciones: Emigrantes chinos y japoneses para las haciendas peruanas. Discover Nikkei, Japanese American National Museum. Los Ángeles, EU. Disponible en: <http://www.discovernikkei.org/ es/journal/2007/9/11/2408/>

Rodríguez Pastor, Humberto (2017). El peón y empresario Nikumatsu Okada y la comunidad japonesa del valle de Chancay (1900-1950). Fondo Editorial de la Asociación Peruano Japonesa, Lima.

Rodríguez Pastor, Humberto (2018). Chinos en la sociedad peruana (1950-2000). Presencia, influencia y alcances. Facultad de Ciencias Sociales de la Universidad Nacional Mayor de San Marcos, Lima.

Sociedad Editorial Panamericana (1924). La colonia china en Perú. Instituciones y hombres representativos. Su actuación benéfica en la vida nacional. Sociedad Panamericana, Lima.

Stewart, Watt (1975). Servidumbre china en el Perú. Ed. Mosca Azul, Lima.

Trazegnies, Fernando (1994). En el país de las colinas de arena. Ts. I y II. Fondo Editorial de la Pontificia Universidad Católica del Perú, Lima. 